\title{
Analytical Model for Heterogeneous Crystallization Kinetics of Spherical Glass Particles
}

\author{
Eduardo Bellini Ferreira, ${ }^{\ddagger, \|}$ Victor Lopez-Richard, ${ }^{\dagger, \S}$ Edgar Dutra Zanotto, and Gilmar Eugenio Marques ${ }^{\S}$ \\ ${ }^{\ddagger}$ Vitrovita Instituto de Inovação em Vitrocerâmicos, 13560-460 São Carlos, SP, Brazil \\ ${ }^{\S}$ Departamento de Física, Universidade Federal de São Carlos, 13.565-905 São Carlos, SP, Brazil
}

`Vitreous Materials Laboratory, Department of Materials Engineering, Universidade Federal de São Carlos, 13.565-905

São Carlos, SP, Brazil

\begin{abstract}
An analytical model developed to describe the crystallization kinetics of spherical glass particles has been derived in this work. A continuous phase transition from three-dimensional (3D)-like to 1D-like crystal growth has been considered and a procedure for the quantitative evaluation of the critical time for this 3D$1 D$ transition is proposed. This model also allows straightforward determination of the density of surface nucleation sites on glass powders using differential scanning calorimetry data obtained under different thermal conditions.
\end{abstract}

\section{Introduction}

MPORTANT surface crystallization related phenomena, such as the vitrification ability of some supercooled liquids and the kinetic competition between viscous flow sintering and surface crystallization of glass powders, ${ }^{1,2}$ are commonly calculated by using an estimated number of heterogeneous nucleation sites per unit surface, $N_{\mathrm{s}}=n /\left(N S_{\mathrm{g}}\right)$. In this equation, it is assumed that a number $N$ of equal-size glass particles each having surface area, $S_{\mathrm{g}}$, can be formed with a total number of crystallization sites, $n$. However, there is no unambiguous and simple method to determine $N_{\mathrm{s}}$ from direct measurements ${ }^{3}$ on small glass particles. The value of $N_{\mathrm{s}}$, the average particle radius, $R$, size distribution and the linear growth rate, $u$, determine the particle crystallization kinetics. ${ }^{4-7}$ In this article, we derived a model to account for the nucleation and growth of crystals of simple geometry on spherical glass particles. This modeling is based on the use of universal parameters and enables a simulation of the crystallization process and all its stages, which vary from three-dimensional (3D) growth on a glass particle surface to $1 \mathrm{D}$ growth toward its interior.

\section{Theoretical Model}

In order to characterize the crystallization of glass particles as a function of annealing time, $t$, we assume the most common case of heterogeneous nucleation of cylindrical crystals growing at a linear rate, $u$, from a fixed number of sites per unit area, $N_{\mathrm{s}}$. Such type of growth generally initiates on the surface of the glass grains, with the circular base growing at the particle surfaces, and the cylindrical axis growing perpendicular to the particles surface toward their interior. According to the Johnson-Mehl-

L. Pinckney—contributing editor

Manuscript No. 26121. Received April 7, 2009; approved June 18, 2009

Author to whom correspondence should be addressed. e-mail: vlopez@df.ufscar.br

"Present address: Engineering Faculty, UNESP, 12.516-410 Guaratinguetá, SP, Brazil.
Avrami-Kolmogorov (JMAK) theory, ${ }^{8-10}$ the crystalline volume fraction of a packing of spherical glass particles of radius $R$ can be given by $^{3}$

$$
\alpha_{\mathrm{v}}\left(t, N_{\mathrm{s}}\right)=1-\exp \left(-\frac{3 \alpha_{\mathrm{s}}\left(t, N_{\mathrm{s}}\right) u t}{R}\right)
$$

(the validity of the JMAK theory has been experimentally confirmed for heterogeneous surface nucleation for the limiting case of large number of nucleation sites ${ }^{11}$ ).

Let us consider a sample with total volume, $V_{\mathrm{T}}$, where the parameter $\alpha_{\mathrm{V}}=V_{\mathrm{c}} / V_{\mathrm{T}}$ characterizes the fraction of $V_{\mathrm{T}}$ that has been crystallized, $V_{\mathrm{c}}$ is the total volume occupied by crystals, $\alpha_{\mathrm{S}}=S_{\mathrm{c}} / S_{\mathrm{g}}$ is the fraction of crystallized surface, defined by the ratio between the crystallized area, $S_{\mathrm{c}}$, and the glass particle surface, $S_{\mathrm{g}}$. The crystals at the surface reduce the effective surface of the glass particles available for further crystallization. Again, according to the JMAK theory, ${ }^{12}$ the crystallized surface fraction is given by

$$
\alpha_{\mathrm{s}}\left(t, N_{\mathrm{s}}\right)=1-\exp \left(-\pi N_{\mathrm{s}} u^{2} t^{2}\right)
$$

Let us now introduce the reduced parameters $\tau \equiv u t / R$ and $v_{\mathrm{s}} \equiv 4 \pi R^{2} N_{\mathrm{s}}$. Note that $v_{\mathrm{s}}$ is the absolute number of nucleation sites on the surface of a spherical glass particle. With these new parameters, Eqs. (1) and (2) can be rewritten as

$$
\alpha_{\mathrm{v}}\left(\tau, v_{\mathrm{s}}\right)=1-\exp \left(-3 \alpha_{\mathrm{s}}\left(\tau, v_{\mathrm{s}}\right) \tau\right)
$$

and

$$
\alpha_{\mathrm{s}}\left(\tau, v_{\mathrm{s}}\right)=1-\exp \left(-\frac{v_{\mathrm{s}} \tau^{2}}{4}\right)
$$

respectively. Thus, the whole kinetic process depends only on dimensionless parameters. The surface and volume time-evolution of crystallization expressed in Eqs. (3) and (4) are universal because they do not explicitly depend on any specific glass composition or temperature. The above equations can be directly linked to differential scanning calorimetric (DSC) measurements.

It has been experimentally confirmed that the area under the DSC crystallization peak of partially crystallized samples, $A\left(\tau, v_{\mathrm{s}}\right)$ (the filled contours in the left panels of Fig. 1) is proportional to the remaining glassy volume:

$$
\alpha_{\mathrm{g}}\left(\tau, v_{\mathrm{s}}\right) V_{\mathrm{T}}=\left(1-\alpha_{\mathrm{v}}\left(\tau, v_{\mathrm{s}}\right)\right) V_{\mathrm{T}}
$$



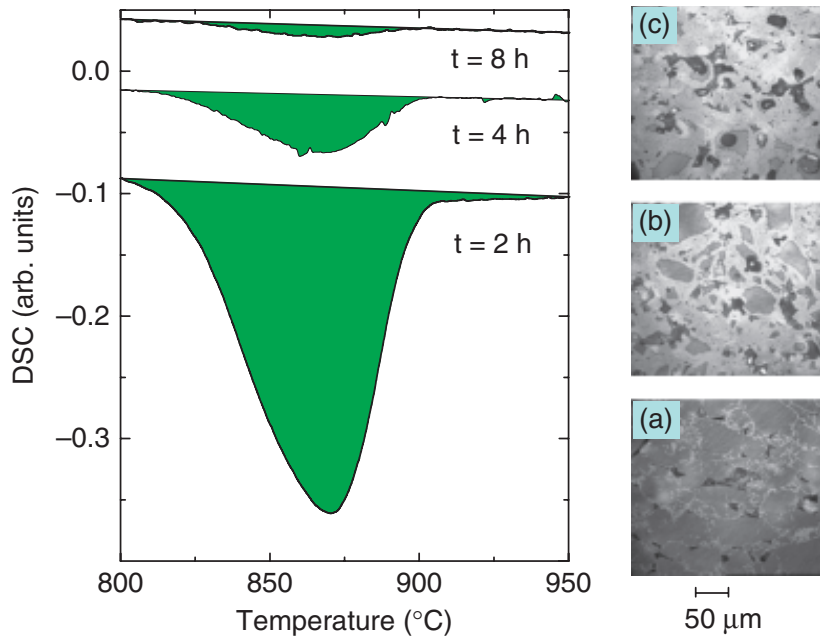

Fig. 1. Right: micrographs of polished sections of diopside glass particles sintered at $825^{\circ} \mathrm{C}$ for: (a) $2 \mathrm{~h}$, (b) $4 \mathrm{~h}$, and (c) $8 \mathrm{~h}$. Color scale: lightgray phase, crystals; medium-dark gray phase, glass; from dark gray to black and eventually white in the center due to reflecting light, pores. Left: the corresponding DSC spectra of samples subjected to the different annealing steps at $825^{\circ} \mathrm{C}$ before the DSC runs.

where $\alpha_{\mathrm{g}}\left(\tau, v_{\mathrm{s}}\right)$ is the glass volume fraction in these samples. (E. B. Ferreira, unpublished data) ${ }^{13,14}$ Thus,

$$
A\left(\tau, v_{\mathrm{s}}\right) \propto V_{\mathrm{T}}\left(1-\alpha_{v}\left(\tau, v_{\mathrm{s}}\right)\right)
$$

The total volume can be expressed by the ratio between the sample mass, $m$, and the density of the solid material as

$$
V_{\mathrm{T}}(\tau)=\frac{m}{\left(\rho_{\mathrm{g}}+\alpha_{\mathrm{v}}\left(\tau, v_{\mathrm{s}}\right)\left(\rho_{\mathrm{c}}-\rho_{\mathrm{g}}\right)\right)}
$$

where $\rho_{\mathrm{c}}$ and $\rho_{\mathrm{g}}$ are the mass densities of the crystal and glass phases, respectively. Now, considering equal-mass samples, it is possible to obtain the ratio between the area under a DSC crystallization peak, at any given time of the crystallization process, $A\left(\tau, v_{\mathrm{s}}\right)$, and the area at $t=0, A\left(0, v_{\mathrm{s}}\right)$ as

$$
\frac{A\left(\tau, v_{\mathrm{s}}\right)}{A\left(0, v_{\mathrm{s}}\right)}=\left(1+\frac{\alpha_{\mathrm{v}}\left(\tau, v_{\mathrm{s}}\right)}{\left(1-\alpha_{\mathrm{v}}\left(\tau, v_{\mathrm{s}}\right)\right)} \frac{\rho_{\mathrm{c}}}{\rho_{\mathrm{g}}}\right)^{-1}
$$

Hence, this equation yields a universal pattern, establishing a direct relationship between the dimensionless parameters $\tau$ and $v_{\mathrm{s}}$, and the ratio between mass densities, $\rho_{\mathrm{c}} / \rho_{\mathrm{g}}$.

\section{Results and Discussion}

In Fig. 1 we show an example of sequential crystal growth steps during the sintering of diopside $\left(\mathrm{CaO} \cdot \mathrm{MgO} \cdot 2 \mathrm{SiO}_{2}\right)$ glass powders. The micrographs correspond to annealing steps performed at $825^{\circ} \mathrm{C}$ for $2 \mathrm{~h} \mathrm{(a),} 4 \mathrm{~h} \mathrm{(b),} \mathrm{and} 8 \mathrm{~h}$ (c). After $2 \mathrm{~h}$ of thermal treatment, crystal formation can be detected around the vitreous grains, shown by light dots in Fig. 1(a). After impingement of the crystals on the surface of the glass grains (Fig. 1(b)), 1D growth prevails, $(t>4 \mathrm{~h})$. This process finally leads to the complete transformation of the former vitreous phase into crystals that grow from the surface toward the grain interior (Fig. 1(c)). These samples were further characterized by DSC, as shown by the corresponding graphics on the left-hand side of Fig. 1.

In Fig. 2(a), the function $A\left(\tau, v_{\mathrm{s}}\right) / A\left(0, v_{\mathrm{s}}\right)$ is displayed for different values of $v_{\mathrm{s}}$, for the ratio $\rho_{\mathrm{c}} / \rho_{\mathrm{g}}=1.162$, where $\rho_{\mathrm{c}}=3261 \mathrm{~kg} / \mathrm{m}^{3}$ and $\rho_{\mathrm{g}}=2805 \mathrm{~kg} / \mathrm{m}^{3}$ (diopside parameters). One can note in this figure the agreement with experimental
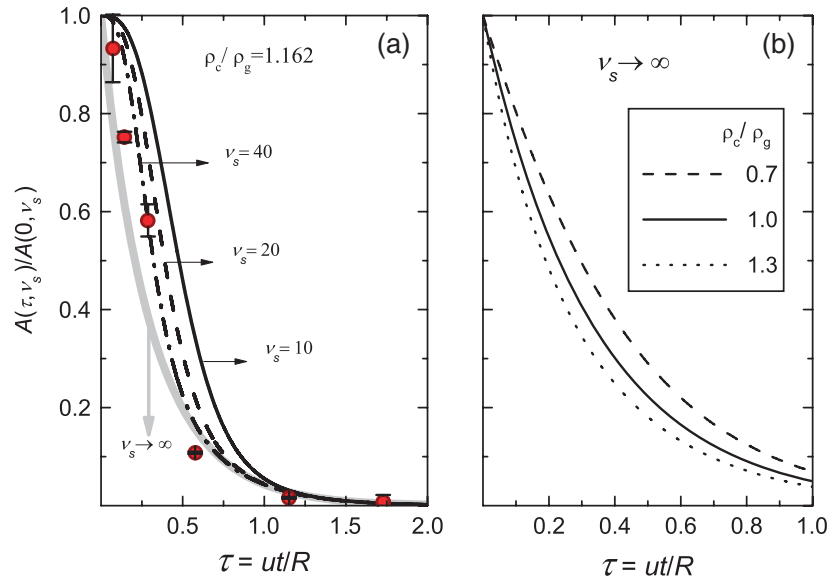

Fig. 2. (a) Plot of $A\left(\tau, v_{\mathrm{s}}\right) / A\left(0, v_{\mathrm{s}}\right)$ for $\rho_{\mathrm{c}} / \rho_{\mathrm{g}}=1.162$ and different values of the universal parameter $v_{\mathrm{s}}$. The limiting case, $v_{\mathrm{s}} \rightarrow \infty$ is indicated by the thick gray solid curve. Circles: experimental points taken from DSC spectra of diopside samples. (b) Plot of $A\left(\tau, v_{\mathrm{s}}\right) / A\left(0, v_{\mathrm{s}}\right)$, in the limiting case $v_{\mathrm{s}} \rightarrow \infty$, for a few values of the ratio $\rho_{\mathrm{c}} / \rho_{\mathrm{g}}$.

points obtained from DSC (some are displayed in Fig. 1), where the nominal values $u=2 \times 10^{-10} \mathrm{~m} / \mathrm{s}$ and $R=5 \mu \mathrm{m}$ were used to obtain the dimensionless parameter $\tau$. For large values of $N_{\mathrm{s}}$ and $v_{\mathrm{s}}$, the crystallization process can be characterized as 1D growth. Such crystal growth conditions are often observed, as shown for example in Fig. 1(b) and (c) for diopside glass samples annealed at $825^{\circ} \mathrm{C}$ for over $4 \mathrm{~h}$, where the surface of the original glass particles is totally crystallized. In the limiting case of large $N_{\mathrm{s}}$ or, according to Eqs. (3) and (4), for $v_{\mathrm{s}} \rightarrow \infty$, the value of the crystalline volume fraction becomes

$$
\alpha_{\mathrm{v}}(\tau, \infty)=1-\exp (-3 \tau)
$$

The corresponding values of $\mathrm{A}(\tau, \infty) / \mathrm{A}(0, \infty)$, for $\rho_{\mathrm{c}} /$ $\rho_{\mathrm{g}}=1.162$, have been indicated in Fig. 2(a) by a thick solid gray curve. They determine the lowest limit of $\mathrm{A}\left(\tau, v_{\mathrm{s}}\right) / \mathrm{A}\left(0, v_{\mathrm{s}}\right)$, for any possible combination of parameters. The dependence on the density ratio $\rho_{\mathrm{c}} / \rho_{\mathrm{g}}$ can be seen in Fig. 2(b), by setting $v_{\mathrm{s}} \rightarrow \infty$ within the interval $\rho_{\mathrm{c}} / \rho_{\mathrm{g}} \in[0.7,1.3]$. Thus, the volume fraction $\alpha_{\mathrm{v}}\left(\tau, v_{\mathrm{s}}\right)$ can be expressed as a function of the area and mass density ratios, $\mathrm{A}\left(\tau, v_{\mathrm{s}}\right) / \mathrm{A}\left(0, v_{\mathrm{s}}\right)$, and $\rho_{\mathrm{c}} / \rho_{\mathrm{g}}$, after using Eq. (8), in the form

$$
\alpha_{\mathrm{v}}\left(\tau, v_{\mathrm{s}}\right)=\left(1+\frac{A\left(\tau, v_{\mathrm{s}}\right) / A\left(0, v_{\mathrm{s}}\right)}{\left(1-A\left(\tau, v_{\mathrm{s}}\right) / A\left(0, v_{\mathrm{s}}\right)\right)} \frac{\rho_{\mathrm{c}}}{\rho_{\mathrm{g}}}\right)^{-1}
$$

One should note that simple DSC measurements performed with two samples with the same mass, a partially crystallized and a glassy one, provide the crystallized volume fraction $\alpha_{\mathrm{v}}\left(\tau, \nu_{\mathrm{s}}\right)$ of materials with known density ratio $\rho_{\mathrm{c}} / \rho_{\mathrm{g}}$. One obtains $\alpha_{\mathrm{v}}\left(\tau, v_{\mathrm{s}}\right)$ from Eq. (10) and by knowing $u$ and $R$, together with Eqs. (3) and (4), one can find the value of the universal parameter $v_{\mathrm{s}}$ as

$$
v_{\mathrm{s}}=-\frac{4}{\tau^{2}} \ln \left(1+\frac{1}{3 \tau} \ln \left(1-\alpha_{\mathrm{v}}\left(\tau, v_{\mathrm{s}}\right)\right)\right)
$$

Equations (10) and (11) show that experimental values of $A\left(\tau, v_{\mathrm{s}}\right) / A\left(0, v_{\mathrm{s}}\right)$ for any $\tau$ and $\rho_{\mathrm{c}} / \rho_{\mathrm{g}}$ determine the universal parameter $v_{\mathrm{s}}$. Consequently, the experimental value for $R$ determines the number of heterogeneous nucleation sites per unit surface, $N_{s}$.

As mentioned before, this surface crystallization process can be divided in two main steps, each one with a predominant morphological characteristic: (i) 3D-like crystal growth on the surface and into the interior of glass particles, (ii) when the total surface 

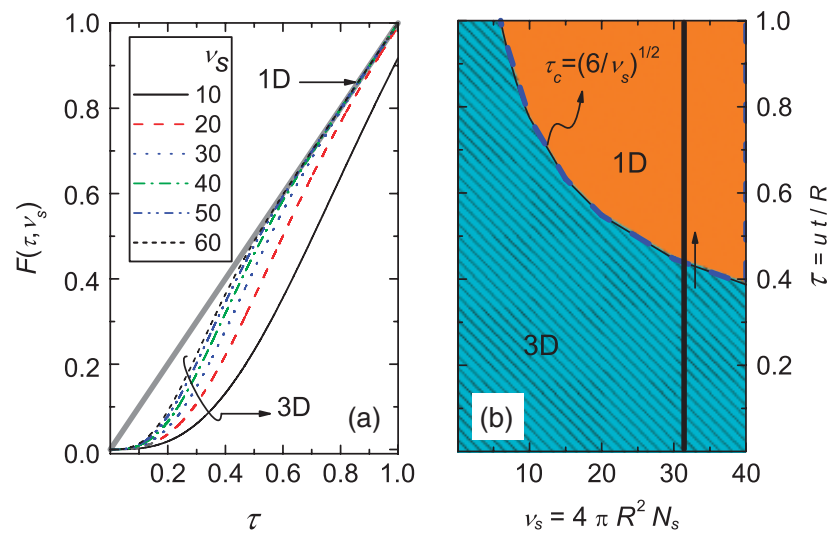

Fig. 3. (a) Function $F\left(\tau, v_{\mathrm{s}}\right)$ for various values of $v_{\mathrm{s}}$ (the asymptotic limit $F\left(\tau, v_{\mathrm{s}}\right) \rightarrow \tau$ that corresponds to the 1D growth mode is shown). (b) Regions of predominant 1D and 3D modes of crystal growth highlighted in continuous and striped patterns, respectively. Dashed curve: calculated critical parameter $\tau_{\mathrm{c}}$, as a function of $v_{\mathrm{s}}$. Solid vertical line: path corresponding to experimental observations shown in Fig. 1.

area of the glass particle is filled with crystals $\left(\alpha_{\mathrm{s}}\left(\tau, \nu_{\mathrm{s}}\right) \rightarrow 1\right)$, the crystal layer continues to grow toward the glass interior in a 1D mode. However, our model allows one to characterize this continuous 3D-1D transition in terms of effective parameters and critical values. We can introduce the function

$$
F\left(\tau, v_{\mathrm{s}}\right) \equiv \frac{1}{3} \ln \left(1-\alpha_{\mathrm{v}}\left(\tau, v_{\mathrm{s}}\right)\right)^{-1}=\alpha_{\mathrm{s}}\left(\tau, v_{\mathrm{s}}\right) \tau
$$

The 3D-like crystal growth is halted when $\alpha_{\mathrm{s}}\left(\tau, v_{\mathrm{s}}\right) \rightarrow 1$ and this condition corresponds to the asymptotic limit $F\left(\tau, v_{\mathrm{s}}\right) \rightarrow \tau$. In Fig. 3(a), we show the plots of $F\left(\tau, v_{\mathrm{s}}\right)$ for different values of $v_{\mathrm{s}}$. In order to reach a criterion for the characterization of the transition between the two crystallization modes, we can define a critical dimensionless time, $\tau_{\mathrm{c}}$, where $d^{2} F\left(\tau_{\mathrm{c}}\right) / \mathrm{d} \tau^{2}=0$. This yields an exact value of the critical dimensionless time for the 3D-1D phase transition between the crystallization growth modes given by $\tau_{\mathrm{c}}=\sqrt{6 / v_{\mathrm{s}}}$ (depicted in Fig. 3(b)). It is important to note that this criterion gives, according to Eq. (4), the boundary value for the crystallized surface fraction

$$
\alpha_{\mathrm{s}}\left(\tau, v_{\mathrm{s}}\right)=1-\exp \left(-\frac{6}{4}\right) \cong 0.7769
$$

which is independent of $v_{\mathrm{s}}$. In terms of the critical dimensionless time, Eq. (4) transforms to

$$
\alpha_{\mathrm{s}}\left(\tau, v_{\mathrm{s}}\right)=1-\exp \left(-\frac{6}{4}\left(\frac{\tau}{\tau_{\mathrm{c}}}\right)^{2}\right)
$$

A universal phase diagram can be produced, as shown in Fig. 3(b), which depends only on $\tau$ and $v_{\mathrm{s}}$ and such a diagram may be used to characterize the crystallization process of spherical glass particles. This is a relevant factor in problems, such as in the control of viscous sintering with concurrent surface crystallization and of the residual porosity in sintered glasses. In the present case, DSC experiments yield a mean value $v_{\mathrm{s}} \sim 30.4$. According to Eq. (11), this corresponds to $N_{\mathrm{s}} \sim 1.2 \times 10^{11} \mathrm{~m}^{-2}$ (the approximate experimental path during the thermal annealing is displayed in Fig. 3(b)). Given that $\tau_{\mathrm{c}}=\sqrt{6 / v_{\mathrm{s}}}$ the value of the critical dimensionless time for diopside yields $\tau_{\mathrm{c}}=0.437$, that corresponds to $3 \mathrm{~h}$ of annealing (note that $\tau \equiv u t / R$ ), in accordance with the micrographs in Fig. 1.

\section{Conclusions}

We developed a universal mathematical treatment to deal with the crystallization kinetics of spherical glass particles. Our model has no boundary when passing from the 3D-like crystallization mode on the surface to a $1 \mathrm{D}$-like mode toward the glass interior. We have shown how the area under DSC crystallization peaks of two samples, one partially crystallized and other glassy, can be used to determine important parameters, such as the number of heterogeneous nucleation sites per unit surface, and the crystallized volume fraction. Finally, we have proposed a phase diagram to characterize the crystallization process of spherical glass particles based on universal parameters.

\section{Acknowledgments}

The authors acknowledge the financial support from the Brazilian agencies FAPESP \# 04/02484-6, \# 07/08179-9, and CNPq. We thank Luis Guilherme Brunetto for conducting some experiments and Vladimir Fokin for a discussion and share of experimental data.

\section{References}

${ }^{1}$ M. L. F. Nascimento, L. A. Souza, E. B. Ferreira, and E. D. Zanotto, "Can Glass Stability Parameters Infer Glass Forming Ability," J. Non-Cryst. Solids., 351, 3296-308 (2005).

${ }^{2}$ M. O. Prado, E. B. Ferreira, and E. D. Zanotto, "Sintering Kinetics of Crystallizing Glass Particles. A Review"; pp. 163-80 in 106th Annual Meeting of The American Ceramic Society Proceedings, Vol. 170, Melt Chemistry, Relaxation, and Solidification Kinetics of Glasses. Ceramic Transactions, Edited by H. Li, C. S. Ray, D. M. Strachan, R. Weber, and Y. Yue. Wiley-Blackwell, Indianapolis, 2005.

${ }^{3}$ E. B. Ferreira, M. L. F. Nascimento, H. Stoppa, and E. D. Zanotto, "Methods to Estimate the Number of Surface Nucleation Sites on Glass Particles," Phys. Chem. Glasses: Eur. J. Glass Sci. Technol. Part B., 49, 81-9 (2008).

${ }^{4}$ M. L. F. Nascimento, E. B. Ferreira, and E. D. Zanotto, "Kinetics and Mechanisms of Crystal Growth and Diffusion in a Glass-Forming Liquid," J. Chem. Phys., 121, 8924-8 (2004).

${ }^{5}$ R. Müller, "The Influence of Grain Size on the Overall Kinetics of SurfaceInduced Glass Crystallization," J. Therm. Anal., 35, 823-35 (1989).

${ }^{6}$ I. Gutzow, R. Pascova, A. Karamanov, and J. Schmelzer, "The Kinetics of Surface Induced Sinter Crystallization and the Formation of Glass-Ceramic Materials," J. Mater. Sci., 33, 5265-73 (1998).

${ }^{7}$ J. Vazquez, D. G.-G. Barreda, P. L. Lopez-Alemany, P. Villares, and R. Jiménez-Garay, "A Comparative Study on the Single-Scan and Multiple-Scan Techniques in Differential Scanning Calorimetry: Application to the Crystallization of the Semiconducting $\mathrm{Ge}_{0.13} \mathrm{Sb}_{0.23} \mathrm{Se}_{0.64}$ Alloy," Thermochim. Acta, 430, 173-82 (2005).

${ }^{8}$ A. Kolmogorov, "On the Statistical Theory of the Crystallization of Metals," Izv. Akad. Nauk SSSR, Ser. Matem., 1, 355-9 (1937).

${ }^{9}$ W. A. Johnson and R. F. Mehl, "Reaction Kinetics in Processes of Nucleation and Growth," Trans. Am. Inst. Min. Metall. Eng., 35, 416-58 (1939).

${ }^{10}$ (a) M. Avrami, "Kinetics of Phase Change. I General Theory," J. Chem. Phys., 7, 1103-12 (1939); (b) M. Avrami, "Kinetics of Phase Change. II Transformation-Time Relations for Random Distribution of Nuclei," J. Chem. Phys., 8 , 212-224 (1940); (c) M. Avrami, "Granulation, Phase Change, and Microstructure Kinetics of Phase Change. III," J. Chem. Phys., 9, 177-184 (1941).

${ }^{11}$ E. D. Zanotto, "Surface Nucleation in a Diopside Glass," J. Non-Cryst. Solids, 130, 217-9 (1991).

${ }^{12}$ J. Farjas and P. Roura, "Numerical Model of Solid Phase Transformations Governed by Nucleation and Growth: Microstructure Development During Isothermal Crystallization," Phys. Rev. B., 75, 184112 (2007).

${ }^{13}$ C. S. Ray, X. Fang, and D. E. Day, "New Method for Determining the Nucleation and Crystal-Growth Rates in Glasses," J. Am. Ceram. Soc., 83, 865-72 (2000)

${ }^{14}$ K. S. Ranasinghe, C. S. Ray, and D. E. Day, "A Generalized Method for Determining the Crystal Nucleation and Growth Rates in Glasses by Differential Thermal Analysis," J. Mater. Sci., 37, 547-55 (2002). 